\title{
Global Finite-Time Output Feedback Stabilization for a Class of Uncertain Nonholonomic Systems
}

\author{
Baojian Du, Fangzheng Gao, and Fushun Yuan \\ School of Mathematics and Statistics, Anyang Normal University, Anyang 455000, China \\ Correspondence should be addressed to Fangzheng Gao; gaofz@126.com
}

Received 7 August 2013; Accepted 3 November 2013

Academic Editor: Daoyi Xu

Copyright (C) 2013 Baojian Du et al. This is an open access article distributed under the Creative Commons Attribution License, which permits unrestricted use, distribution, and reproduction in any medium, provided the original work is properly cited.

\begin{abstract}
This paper investigates the problem of global finite-time stabilization by output feedback for a class of nonholonomic systems in chained form with uncertainties. By using backstepping recursive technique and the homogeneous domination approach, a constructive design procedure for output feedback control is given. Together with a novel switching control strategy, the designed controller renders that the states of closed-loop system are regulated to zero in a finite time. A simulation example is provided to illustrate the effectiveness of the proposed approach.
\end{abstract}

\section{Introduction}

Over the past decade, nonholonomic systems have attracted much attention because they can be used to model many real systems, such as mobile robots, car-like vehicle, and underactuated satellites. An important feature of a nonholonomic system is that the number of its inputs is less than the number of its degree of freedom, which makes the control problems of a nonholonomic system challenging. As pointed out by Brockett in [1], there does not exist a pure-state feedback control law for a nonholonomic system such that its state converges to its equilibrium. To overcome this difficulty, with the effort of many researchers a number of intelligent approaches have been proposed, which can be classified into discontinuous control laws [2, 3], time-varying control laws [4-6], and hybrid control laws [7, 8]; see the survey paper [9] for more details and references therein. Considering the difficulty of measuring full states and the inevitability of uncertainties in engineering practice, the output feedback issue of nonholonomic systems with drift uncertainties has recently been studied [10-16]. However, it should be mentioned that the aforementioned works only consider the feedback stabilizer that makes the trajectories of the systems converge to the equilibrium as the time goes to infinity.

Compared to the asymptotic stabilization, the finite-time stabilization, which renders the trajectories of the closed-loop systems convergent to the origin in a finite time, has many advantages such as fast response, high tracking precision, and disturbance-rejection properties [17]. Hence, it is more meaningful to investigate the finite-time stabilization problem than the classical asymptotical stability. In recent years, the problem of finite-time stabilization for nonlinear systems has been studied and some interesting results have been obtained [1825]. However, the finite-time stabilization of nonholonomic systems is a relatively new problem. In fact, even in the case of finite-time stabilization using state feedback, there are very few results in the literature [26-28]. In the case when parts of the states are not measurable, to stabilize a nonholonomic system in a finite time only using limited measurable states becomes challenging.

To illustrate the difficulties in finite-time control of nonholonomic systems via output feedback, let us consider a problem of finite time stabilizing the following simple system at the origin:

$$
\dot{x}_{0}=u_{0}, \quad \dot{x}_{1}=x_{1} u_{0}, \quad \dot{x}_{2}=u_{1},
$$

where $x_{0}$ and $x_{1}$ are measurable and $x_{2}$ is not available for feedback.

In discontinuous approach, as seen, for example, in [2628 ], assuming that $x_{0}\left(t_{0}\right) \neq 0$ one might design the control $u_{0}$ as follows:

$$
u_{0}\left(x_{0}\right)=-k_{0} x_{0}^{\alpha_{0}}, \quad 0<\alpha_{0}=\frac{\alpha_{1}}{\alpha_{2}}<1
$$


where $k_{0}$ is a positive design parameter and $\alpha_{i}, i=1,2$, are positive odd numbers. It is easy to verify that the $u_{0}$ in (2) renders $x_{0}$ globally converging to zero in a finite time $T_{0}$.

Next, we need to stabilize the $x$-subsystem

$$
\dot{x}_{1}=-k_{0} x_{0}^{\alpha_{0}} x_{2}, \quad \dot{x}_{2}=u_{1}
$$

within a settling time $T_{1}$ satisfying $T_{1}<T_{0}$. By introducing the input-state-scaling transformation $x_{1}=x_{1} / u_{0}$ and $z_{2}=$ $x_{2}$, the system (3) can be rewritten as

$$
\dot{z}_{1}=z_{2}+\frac{\dot{u}_{0}}{u_{0}} z_{1}, \quad \dot{z}_{2}=u_{1} .
$$

However, the system (3) possesses the time-varying coefficient $-k_{0} x_{0}^{\alpha_{0}}$ (or the system (4) dissatisfies the low-order growth condition), which renders the existing finite-time control methods highly difficult to the control problem of the $x$-subsystem or even inapplicable. To the best of the authors' knowledge, there is no result referred to the finite-time stabilization of nonholonomic systems by output feedback.

Motivated by the aforementioned discussion, in this paper we aim to tackle this challenging question and provide a solution to the problem of global finite-time output feedback stabilization for nonholonomic systems with uncertainties by applying the homogeneous domination approach. The main contribution of this paper is twofold. (i) Compared to the existing output feedback stabilization results for nonholonomic systems, the finite-time stabilizer proposed in this paper leads to faster convergence rate. (ii) As the common assumption to guarantee the existence of global finite-time output feedback stabilizer for a nonlinear system, the loworder growth (the order less than one) of system nonlinearities renders the discontinuous change of coordinates (i.e., the $\sigma$-process) inapplicable to the finite-time output feedback control problem of the nonholonomic systems, even the ideal chained systems, and how to deal with this constitutes one of the main contributions of this paper.

The rest of this paper is organized as follows. Section 2 provides the problem formation and preliminary knowledge. Section 3 presents the control design procedure and the main result, while Section 4 gives a simulation example to illustrate the theoretical finding of this paper. Finally, concluding remarks are proposed in Section 5.

\section{Problem Formulation and Preliminaries}

In this paper, we consider the following uncertain nonholonomic systems:

$$
\begin{gathered}
\dot{x}_{0}=d_{0} u_{0}+\phi_{0}\left(t, x_{0}\right), \\
\dot{x}_{i}=d_{i} x_{i+1} u_{0}+\phi_{i}\left(t, x_{0}, x\right), \quad i=1, \ldots, n-1, \\
\dot{x}_{n}=d_{n} u_{1}+\phi_{n}\left(t, x_{0}, x\right), \\
y=\left(x_{0}, x_{1}\right)^{T},
\end{gathered}
$$

where $\left(x_{0}, x\right)^{T}=\left(x_{0}, x_{1}, \ldots, x_{n}\right)^{T} \in R^{n+1}, u=\left(u_{0}, u_{1}\right)^{T} \in R^{2}$, $y \in R^{2}$ are the system state, control input, and system output, respectively, $d_{i}$ 's are disturbed virtual control coefficients, and $\phi_{i}$ 's denote the input and states driven uncertainties, which are called the nonlinear drifts of the system (5).

The objective of this paper is to design an output feedback controller in the form

$$
\dot{\hat{x}}=\vartheta(\widehat{x}, y), \quad u_{0}=u_{0}\left(x_{0}\right), \quad u_{1}=u_{1}(\hat{x}, y),
$$

such that the finite-time regulation of the states is achieved; that is, $\lim _{t \rightarrow T}\left(\left|x_{0}(t)\right|+|x(t)|\right)=0$ and $\left(x_{0}(t), x(t)\right)=(0,0)$ for any $t \geq T$, where $T$ is a finite settling time.

To this end, the following assumptions regarding system (5) are imposed.

Assumption 1. For $i=0,1, \ldots, n$, there are positive constants $c_{i 1}$ and $c_{i 2}$ such that

$$
c_{i 1} \leq d_{i} \leq c_{i 2} .
$$

Assumption 2. For $\phi_{0}$, there is a positive constant $a$ such that

$$
\left|\phi_{0}\left(t, x_{0}\right)\right| \leq a\left|x_{0}\right|
$$

Assumption 3. For $i=1, \ldots, n$, there are constants $b>0$ and $\tau \in(-1 / n, 0)$ such that

$$
\left|\phi_{i}\left(t, x_{0}, x\right)\right| \leq b\left(\left|x_{1}\right|^{\left(r_{i}+\tau\right) / r_{1}}+\cdots+\left|x_{i}\right|^{\left(r_{i}+\tau\right) / r_{i}}\right),
$$

where $r_{i}=1+(i-1) \tau$.

For simplicity, in this paper we assume $\tau=-p / q$ with $p$ being any even integer and $q$ being any odd integer. Based on this, we know that $r_{i} \in(0,1)$ is a ratio of two positive odd integers.

Remark 4. Assumptions 1-2 are common and similar to the one usually imposed on the nonlinear systems [10]. Relatively speaking, Assumption 3 seems to be quite restrictive; however, it plays an essential role in ensuring the existence of finite-time output feedback stabilizer for nonholonomic system (5). Furthermore, it is worth pointing out that there are a number of nonlinear functions such as $\sin x$ and $\ln \left(1+x^{2}\right)$ that can be bounded by a function $|x|^{m}$ for any constant $m \in(0,1)$ actually satisfying this assumption.

The following definitions and lemmas will serve as the basis of the coming control design and performance analysis.

Definition 5 (see [17]). Consider a system

$$
\dot{x}=f(x) \text { with } f(0)=0, x \in R^{n},
$$

where $f: U_{0} \rightarrow R^{n}$ is continuous with respect to $x$ on an open neighborhood $U_{0}$ of the origin $x=0$. The equilibrium $x=0$ of the system is (locally) finite-time stable if it is Lyapunov stable and finite-time convergent in a neighborhood $U \in U_{0}$ of the origin. By "finite-time convergence," we mean that if, for any initial condition, $x(0) \in U$, there is a settling time $T>0$, such that every solution $x(t)$ with $x(0)$ as its initial condition of (10) is well defined with $x(0) \in U \backslash\{0\}$ for $t \epsilon$ $[0, T)$ and satisfies $\lim _{t \rightarrow T} x(t)=0$ and $x(t)=0$ for any $t \geq T$. If $U=U_{0}=R^{n}$, the origin is a globally finite-time stable equilibrium. 
Lemma 6 (see [17]). Consider the nonlinear system described in (10). Suppose that there is a $C^{1}$ function $V(x)$ defined in a neighborhood $\widehat{U} \in R^{n}$ of the origin, real numbers $c>0$, and $0<\alpha<1$, such that

(i) $V(x)$ is positive definite on $\widehat{U}$;

(ii) $\dot{V}(x)+c V^{\alpha}(x) \leq 0, \forall x \in \widehat{U}$.

Then, the origin of system (10) is locally finite-time stable with

$$
T \leq \frac{V^{1-\alpha}(x(0))}{c(1-\alpha)}
$$

for initial condition $x(0)$ in some open neighborhood $U \in \widehat{U}$ of the origin. If $U=R^{n}$ and $V(x)$ is also radially unbounded (i.e., $V(x) \rightarrow+\infty$ as $x \rightarrow+\infty$ ), the origin of system (10) is globally finite-time stable.

Definition 7 (see [29]). Weighted homogeneity: for fixed coordinates $\left(x_{1}, \ldots, x_{n}\right) \in R^{n}$ and real numbers $r_{i}>0$, $i=1, \ldots, n$, one has the following.

(i) The dilation $\Delta_{\varepsilon}(x)$ is defined by $\Delta_{\varepsilon}(x)=\left(\varepsilon^{r_{1}} x_{1}, \ldots\right.$, $\varepsilon^{r_{n}} x_{n}$ ) for any $\varepsilon>0$, where $r_{i}$ is called the weights of the coordinates. For simplicity, we define dilation weight $\Delta=\left(r_{1}, \ldots, r_{n}\right)$.

(ii) A function $V \in\left(R^{n}, R\right)$ is said to be homogeneous of degree $\tau$ if there is a real number $\tau \in R$ such that $V\left(\Delta_{\varepsilon}(x)\right)=\varepsilon^{\tau} V\left(x_{1}, \ldots, x_{n}\right)$ for any $x \in R^{n} \backslash\{0\}, \varepsilon>0$.

(iii) A vector field $f \in\left(R^{n}, R^{n}\right)$ is said to be homogeneous of degree $\tau$ if there is a real number $\tau \in R$ such that $f_{i}\left(\Delta_{\varepsilon}(x)\right)=\varepsilon^{\tau+r_{i}} f_{i}(x)$, for any $x \in R^{n} \backslash\{0\}, \varepsilon>0$, $i=1, \ldots, n$.

(iv) A homogeneous $p$-norm is defined as $\|x\|_{\Delta, p}=$ $\left(\sum_{i=1}^{n}\left|x_{i}\right|^{p / r_{i}}\right)^{1 / p}$ for all $x \in R^{n}$, for a constant $p \geq 1$. For simplicity, in this paper, one chooses $p=2$ and writes $\|x\|_{\Delta}$ for $\|x\|_{\Delta, 2}$.

Lemma 8 (see [30]). Suppose that $V: R^{n} \rightarrow R$ is a homogeneous function of degree $\tau$ with respect to the dilation weight $\Delta$. Then the following hold.

(i) $\partial V / \partial x_{i}$ is homogeneous of degree $\tau-r_{i}$ with $r_{i}$ being the homogeneous weight of $x_{i}$.

(ii) There is a constantc such that $V(x) \leq c\|x\|_{\Delta}^{\tau}$. Moreover, if $V(x)$ is positive definite, then $\underline{c}\|x\|_{\Delta}^{\tau} \leq V(x)$, where $\underline{c}$ is a constant.

Lemma 9 (see [31]). For $x \in R, y \in R$, and $p \geq 1$ which is a constant, the following inequalities hold:

$$
\begin{gathered}
|x+y|^{p} \leq 2^{p-1}\left|x^{p}+y^{p}\right| \\
(|x|+|y|)^{1 / p} \leq|x|^{1 / p}+|y|^{1 / p} \leq 2^{(p-1) / p}(|x|+|y|)^{1 / p} .
\end{gathered}
$$

If $p \geq 1$ is odd, then

$$
\begin{gathered}
|x-y|^{p} \leq 2^{p-1}\left|x^{p}-y^{p}\right|, \\
\left|x^{1 / p}-y^{1 / p}\right| \leq 2^{(p-1) / p}(|x-y|)^{1 / p} .
\end{gathered}
$$

Lemma 10 (see [32]). Let $x, y$ be real variables; then for any positive real numbers $a, m$, and $n$, one has

$$
\begin{aligned}
& a|x|^{m}|y|^{n} \\
& \quad \leq b|x|^{m+n}+\frac{n}{m+n}\left(\frac{m+n}{m}\right)^{-m / n} a^{(m+n) / n} b^{-m / n}|y|^{m+n},
\end{aligned}
$$

where $b>0$ is any real number.

Lemma 11 (see [33]). For $x, y \in R$ and positive real number $p$, the following inequality holds:

$$
\begin{aligned}
\left|x^{p}-y^{p}\right| & \leq p|x-y|\left|x^{p-1}+y^{p-1}\right| \\
& \leq c|x-y|\left|(x-y)^{p-1}+y^{p-1}\right|,
\end{aligned}
$$

where $c=p$ for $1<p \leq 2$ and $c=p 2^{p-1}$ for $p>2$.

\section{Finite-Time Output Feedback Controller Design}

In this section, we give a constructive procedure for the finitetime stabilizer of system (5) by output feedback. The design of finite-time output feedback controller is divided into the following two steps.

(i) We first stabilize the $x$-subsystem in a finite time by output feedback.

(ii) Then we design a controller such that the $x_{0}$-subsystem is finite-time stable.

3.1. Finite-Time Output Feedback Stabilization of the $x$-Subsystem. For the $x_{0}$-subsystem, we choose the control $u_{0}$ as

$$
u_{0} \equiv u_{0}^{*},
$$

where $u_{0}^{*}$ is a positive constant. In this case, the $x_{0}$-subsystem becomes

$$
\dot{x}_{0}=d_{0} u_{0}^{*}+\phi_{0}\left(t, x_{0}\right) \text {. }
$$

Noting that $\phi_{0}\left(t, x_{0}\right)$ satisfies the linear growth condition, it is easy to obtain that the solution of $x_{0}$-subsystem is bounded, for any given finite time $t_{s}>0$. Hence, $x_{0}$ is well defined on $\left[0, t_{s}\right]$. Under the control law (16), the $x$-subsystem can be written as

$$
\begin{gathered}
\dot{x}_{i}=d_{i} u_{0}^{*} x_{i+1}+\phi_{i}\left(t, x_{0}, x\right), \quad i=1, \ldots, n-1, \\
\dot{x}_{n}=d_{n} u_{1}+\phi_{n}\left(t, x_{0}, x\right) .
\end{gathered}
$$

Next we consider the finite-time output feedback stabilizer for system (18). For convenience, we define the following change of coordinates:

$$
\zeta_{1}=x_{1}, \quad \zeta_{i}=d_{1} \cdots d_{i-1} u_{0}^{* i-1} x_{i}, \quad i=2, \ldots, n
$$


under which system (18) is transformed into

$$
\begin{gathered}
\dot{\zeta}_{i}=\zeta_{i+1}+f_{i}\left(t, x_{0}, \zeta\right), \quad i=1, \ldots, n-1, \\
\dot{\zeta}_{n}=d u_{1}+f_{n}\left(t, x_{0}, \zeta\right),
\end{gathered}
$$

where $d=d_{1} \cdots d_{n} u_{0}^{* n-1}, f_{i}=d_{1} \cdots d_{i-1} u_{0}^{* i-1} \phi_{i}$, and the state $\zeta_{1}=x_{1}$ is measurable.

Remark 12. It is worth pointing out that, in terms of the transformation (19), the stabilizing control design of system (18) is equivalent to that of system (20). Thus, in what follows, we turn to designing the output feedback stabilizing controller for system (20) rather than (18). Moreover, with the help of Assumptions 1 and 3 , it can be verified that $f_{i}, i=1, \ldots, n$, satisfy

$$
\left|f_{i}\left(t, x_{0}, \zeta\right)\right| \leq b\left(\left|\zeta_{1}\right|^{\left(r_{i}+\tau\right) / r_{1}}+\cdots+\left|\zeta_{i}\right|^{\left(r_{i}+\tau\right) / r_{i}}\right)
$$

with a new growth rate $b$.

To construct a global output feedback controller for system (20), we will employ the homogeneous domination approach introduced in [34]. We will first construct specifically a homogeneous output feedback controller for the nominal system without considering perturbing terms $f_{i}$ 's. Then, we utilize a scaling gain in the controller to dominate the uncertain nonlinearities $f_{i}$ 's.

3.1.1. Homogeneous Output Feedback Control of the Nominal System. In this subsection, we will construct an output feedback stabilizer for the following nominal system:

$$
\dot{z}_{i}=z_{i+1}, \quad i=1, \ldots, n-1, \quad \dot{z}_{n}=d v .
$$

The design of output feedback controller is divided into two steps. In Step A, we suppose that all the states are measurable and develop a recursive design method to explicitly construct a state feedback control law for system (22). Then in Step B, by constructing a nonsmooth reduced-order observer, we design an output feedback controller.

\section{(A) State Feedback Controller Design}

Step 1. Choose the Lyapunov function $V_{1}=x_{1}^{2} / 2$. Clearly, the first virtual controller

$$
z_{2}^{*}=-n z_{1}^{r_{2}}:=-\beta_{1} \xi_{1}^{r_{2}}
$$

with $\xi_{1}=z_{1}$ and $\beta_{1}=n$ renders

$$
\dot{V}_{1} \leq-n \xi_{1}^{2+\tau}+\xi_{1}\left(z_{2}-z_{2}^{*}\right) \text {. }
$$

Step $i(2 \leq i \leq n)$. In this step, we can obtain the following property.

Proposition 13. For the ith Lyapunov function defined by

$$
V_{i}=V_{i-1}+\int_{z_{i}^{*}}^{z_{i}}\left(s^{1 / r_{i}}-z_{i}^{* 1 / r_{i}}\right)^{2-r_{i}} d s
$$

under the coordinate transformation

$$
z_{k}^{*}=-\beta_{k-1} \xi_{k-1}^{r_{k}}, \quad \xi_{k}=z_{k}^{1 / r_{k}}-z_{k}^{* 1 / r_{k}}, \quad k=2, \ldots, i
$$

there exists the $C^{0}$ virtual controller $z_{i+1}^{*}=-\beta_{i} \xi_{i}^{1 / r_{i+1}}$ such that

$$
\dot{V}_{i} \leq-(n-i+1)\left(\xi_{1}^{2+\tau}+\cdots+\xi_{i}^{2+\tau}\right)+\xi_{i}^{2-r_{i}}\left(z_{i+1}-z_{i+1}^{*}\right),
$$

where $\beta_{j}>0, j=1, \ldots, i$ are constants.

Proof. The detailed proof can be found in [20] and hence is omitted here.

From the inductive steps, we can design

$$
\begin{aligned}
z_{n+1}^{*}= & -\beta_{n} \xi_{n}^{r_{n}+\tau} \\
=- & \beta_{n}\left(z_{n}^{1 / r_{n}}+\beta_{n-1}^{1 / r_{n}}\right. \\
& \left.\quad \times\left(z_{n-1}^{1 / r_{n-1}}+\cdots+\beta_{2}^{1 / r_{3}}\left(z_{2}^{1 / r_{2}}+\beta_{1}^{1 / r_{2}} z_{1}\right)\right)\right)^{r_{n}+\tau} \\
& =-\beta_{n}\left(\bar{\beta}_{n} z_{n}^{1 / r_{n}}+\bar{\beta}_{n-1} z_{n-1}^{1 / r_{n-1}}+\cdots+\bar{\beta}_{1} z_{1}\right)^{r_{n}+\tau},
\end{aligned}
$$

where

$$
\bar{\beta}_{i}= \begin{cases}\beta_{n-1}^{1 / r_{n}} \cdots \beta_{i}^{1 / r_{i+1}}, & i=1, \ldots, n-1 \\ 1, & i=n\end{cases}
$$

such that

$$
\dot{V}_{n} \leq-\left(\xi_{1}^{2+\tau}+\cdots+\xi_{n}^{2+\tau}\right)+d \xi_{n}^{2-r_{n}}\left(v-z_{n+1}^{*}\right) .
$$

(B) Output Feedback Controller Design. Since $z_{2}, \ldots, z_{n}$ are unmeasurable, we construct a homogeneous observer

$$
\dot{\eta}_{i}=-l_{i-1} \widehat{z}_{i}, \quad \widehat{z}_{i}=\left(\eta_{i}+l_{i-1} \widehat{z}_{i-1}\right)^{r_{i} / r_{i-1}}, \quad i=2, \ldots, n,
$$

where $\widehat{z}_{1}=z_{1}$ and $l_{i}>0 ; i=1, \ldots, n-1$ are the gains to be determined. By the certainty equivalence principle, we can replace $z_{i}$ with $\widehat{z}_{i}$ in (28) and obtain an output feedback controller

$$
v(\widehat{z})=-\beta_{n}\left(\bar{\beta}_{n} \widehat{z}_{n}^{1 / r_{n}}+\bar{\beta}_{n-1} \widehat{z}_{n-1}^{1 / r_{n-1}}+\cdots+\bar{\beta}_{1} z_{1}\right)^{r_{n}+\tau},
$$

where $\widehat{z}=\left(z_{1}, \widehat{z}_{2}, \ldots, \widehat{z}_{n}\right)$.

Considering

$$
W_{i}=\int_{\gamma_{i}^{\left(2-r_{i-1}\right) / r_{i-1}}}^{z_{i}^{\left(2-r_{i-1}\right) / r_{i}}}\left(s^{r_{i-1} /\left(2-r_{i-1}\right)}-\gamma_{i}\right) d s,
$$

where $\gamma_{i}=\eta_{i}+l_{i-1} z_{i-1}$, and setting $e_{i}=\left(z_{i}-\widehat{z}_{i}\right)^{1 / r_{i}}$, for $i=$ $2, \ldots, n$, from (22), (31), and (33), it follows that

$$
\begin{aligned}
\dot{W}_{i}= & \frac{2}{r_{i}} r_{i-1} z_{i}^{\left(2-r_{i-1}-r_{i}\right) / r_{i}}\left(z_{i}^{r_{i-1} / r_{i}}-\gamma_{i}\right) z_{i+1} \\
& -l_{i-1} e_{i}^{r_{i}}\left(z_{i}^{\left(2-r_{i-1}\right) / r_{i}}-\widehat{z}_{i}^{\left(2-r_{i-1}\right) / r_{i}}\right) \\
& -l_{i-1} e_{i}^{r_{i}}\left(\widehat{z}_{i}^{\left(2-r_{i-1}\right) / r_{i}}-\gamma_{i}^{\left(2-r_{i-1}\right) / r_{i-1}}\right),
\end{aligned}
$$

where $z_{n+1}=v(\widehat{z})$. 
Each term on the right-hand side of (34) can be estimated by the following propositions whose proofs are given in the Appendix.

Proposition 14. There exists a positive constant $\lambda_{i}$ such that

$$
-l_{i-1} e_{i}^{r_{i}}\left(z_{i}^{\left(2-r_{i-1}\right) / r_{i}}-\widehat{z}_{i}^{\left(2-r_{i-1}\right) / r_{i}}\right) \leq-l_{i-1} \lambda_{i} e_{i}^{2+\tau} .
$$

Proposition 15. For $i=2, \ldots, n-1$,

$$
\begin{aligned}
& \frac{2-r_{i-1}}{r_{i}} z_{i}^{\left(2-r_{i-1}-r_{i}\right) / r_{i}}\left(z_{i}^{r_{i-1} / r_{i}}-\gamma_{i}\right) z_{i+1} \\
& \quad \leq \frac{1}{12} \sum_{j=i-1}^{i+1} \xi_{j}^{2+\tau}+m_{i} e_{i}^{2+\tau}+g_{i}\left(l_{i-1}\right) e_{i-1}^{2+\tau},
\end{aligned}
$$

where $g_{i}$ is a continuous function of $l_{i-1}, m_{i}>0$ is a constant, and $g_{2}=0$.

Proposition 16. For the controller $v(\widehat{z})$, one obtains

$$
\begin{gathered}
\frac{2-r_{n-1}}{r_{n}} z_{n}^{\left(2-r_{n-1}-r_{n}\right) / r_{n}}\left(z_{n}^{r_{n-1} / r_{n}}-\gamma_{n}\right) v(\widehat{z}) \\
\leq \frac{1}{8} \sum_{j=1}^{n} \xi_{j}^{2+\tau}+c \sum_{i=2}^{n} e_{i}^{2+\tau}+g_{n}\left(l_{n-1}\right) e_{n-1}^{2+\tau},
\end{gathered}
$$

where $g_{n}$ is a continuous function of $l_{n-1}$ and $c>0$ is a constant.

Proposition 17. For $i=3, \ldots, n$,

$$
\begin{aligned}
& -l_{i-1} e_{i}^{r_{i}}\left(\widehat{z}_{i}^{\left(2-r_{i-1}\right) / r_{i}}-\gamma_{i}^{\left(2-r_{i-1}\right) / r_{i-1}}\right) \\
& \quad \leq \frac{1}{16}\left(\xi_{i-1}^{2+\tau}+\xi_{i}^{2+\tau}\right)+e_{i}^{2+\tau}+h_{i}\left(l_{i-1}\right) e_{i-1}^{2+\tau},
\end{aligned}
$$

where $h_{i}$ is a continuous function of $l_{i-1}$.

Choosing $W=\sum_{i=2}^{n} W_{i}$, by Propositions 14-17, we get

$$
\begin{aligned}
\dot{W}= & \frac{1}{2} \sum_{i=1}^{n} \xi_{i}^{2+\tau}+\left(-l_{1} \lambda_{2}+m_{2}+c+g_{3}\left(l_{2}\right)+h_{3}\left(l_{2}\right)\right) e_{2}^{2+\tau} \\
& +\sum_{i=3}^{n-1}\left(-l_{i-1} \lambda_{i}+m_{i}+1+c+g_{i+1}\left(l_{i}\right)+h_{i+1}\left(l_{i}\right)\right) e_{i}^{2+\tau} \\
& +\left(-l_{n-1} \lambda_{n}+1+c\right) e_{n}^{2+\tau} .
\end{aligned}
$$

By (28), (32), and Assumption 1, we can estimate $d \xi_{i}^{2-r_{n}}\left(v-z_{n+1}^{*}\right)$ in (29) by the following proposition, whose proof is given in the Appendix.

Proposition 18. There exists a positive constant $\mu$ such that

$$
d \xi_{i}^{2-r_{n}}\left(v-z_{n+1}^{*}\right) \leq \frac{1}{4} \sum_{i=1}^{n} \xi_{i}^{2+\tau}+\mu \sum_{i=2}^{n} e_{i}^{2+\tau}
$$

where $g_{n}$ is a continuous function of $l_{n-1}$.
With the help of Proposition 18, defining $U=V_{n}+W$, combining (29) and (39), and recursively choosing

$$
\begin{aligned}
& l_{n-1} \geq \lambda_{n}^{-1}\left(\frac{1}{4}+1+c+\mu\right), \\
& l_{i-1} \geq \lambda_{i}^{-1}\left(\frac{1}{4}+m_{i}+1+c+\mu+g_{i+1}\left(l_{i}\right)+h_{i+1}\left(l_{i}\right)\right) \\
& i=n-1, \ldots, 3, \\
& \quad l_{1} \geq \lambda_{2}^{-1}\left(\frac{1}{4}+m_{2}+c+\mu+g_{3}\left(l_{2}\right)+h_{3}\left(l_{2}\right)\right)
\end{aligned}
$$

we obtain

$$
\dot{U}=-\frac{1}{4} \sum_{i=1}^{n} \xi_{i}^{2+\tau}-\frac{1}{4} \sum_{i=2}^{n} e_{i}^{2+\tau}
$$

Since $U$ is positive definite and proper with respect to $Z=$ $\left(z_{1}, \ldots, z_{n}, \eta_{2} \ldots, \eta_{n}\right)^{T},(42)$ implies that the closed-loop system can be rewritten as the following compact form:

$$
\dot{Z}=F(Z)=\left(z_{2}, \ldots, z_{n}, d v, \dot{\eta}_{2} \ldots, \dot{\eta}_{n}\right)^{T}
$$

which is homogeneous with the dilation weight

$$
\begin{aligned}
\Delta & =(\underbrace{r_{1}, \ldots, r_{n}}_{\text {for } z_{1}, \ldots, z_{n}}, \underbrace{r_{1}, \ldots, r_{n-1}}_{\text {for } \eta_{2} \ldots, \eta_{n}}) \\
& =(\underbrace{1, \ldots, 1+(n-1) \tau}_{\text {for } z_{1}, \ldots, z_{n}}, \underbrace{1, \ldots, 1+(n-2) \tau}_{\text {for } \eta_{2}, \ldots, \eta_{n}}) .
\end{aligned}
$$

It can be shown that (43) is homogeneous of degree $\tau$. In addition, $U$ is homogeneous of degree 2 . By Lemma 8 , there is a constant $\bar{c}_{1}$, such that

$$
U \leq \bar{c}_{1}\|Z\|_{\Delta}^{2}
$$

where $\bar{c}_{1}>0$ and $\|Z\|_{\Delta}=\sqrt{\left(\sum_{i=1}^{2 n-1}\left|Z_{i}\right|^{2 / r_{i}}\right)}$. Similarly, since the right-hand side of (42) is homogeneous of degree $2+\tau$, by Lemma 8 there is a constant $\bar{c}_{2}$ such that

$$
\frac{\partial U}{\partial Z} F(Z) \leq-\frac{1}{4} \sum_{i=1}^{n} \xi_{i}^{2+\tau}-\frac{1}{4} \sum_{i=2}^{n} e_{i}^{2+\tau} \leq-\bar{c}_{2}\|Z\|_{\Delta}^{2+\tau} .
$$

Combining (45) and (46), it can be deduced from (42) that

$$
\dot{U} \leq-k U^{(2+\tau) / 2}
$$

for a constant $k>0$. By Lemma 6 with $\alpha=(2+\tau) / 2<1$, the closed-loop system is globally finite-time stable.

Remark 19. It should be pointed out that the output feedback controller (32) is only continuous (rather than continuously differentiable) due to the presence of the powers $r_{n}+\tau$, which is less than one. As a consequence, the closed-loop system (22) and (32) is not locally Lipschitz. Therefore, the uniqueness of the solution of system (22) and (32) is not guaranteed. Fortunately, as shown in the work [35], the existence of the solution can still be guaranteed for a continuous system without Lipschitz condition. 
3.1.2. Homogeneous Output Feedback Control of the System (20). Together with the homogeneous controller and observer established previously, in this subsection we are ready to use the homogeneous domination approach to globally stabilize (20) via output feedback under (21). First, we introduce the change of coordinates

$$
\begin{gathered}
z_{i}=\frac{\eta_{i}}{L^{i-1}}, \quad i=2, \ldots, n, \\
v=\frac{u_{1}}{L^{n}},
\end{gathered}
$$

where $L \geq 1$ is a constant to be determined later. Under (48), system (20) can be rewritten as

$$
\begin{gathered}
\dot{z}_{i}=L z_{i+1}+\frac{f_{i}(\cdot)}{L^{i-1}}, \quad i=1, \ldots, n-1, \\
\dot{z}_{n}=L d v++\frac{f_{n}(\cdot)}{L^{n-1}} .
\end{gathered}
$$

Now we construct an observer with a gain $L$ as follows:

$$
\dot{\eta}_{i}=-L l_{i} \widehat{z}_{i}, \quad \widehat{z}_{i}=\left(\eta_{i}+l_{i-1} \widehat{z}_{i-1}\right)^{r_{i} / r_{i-1}}, \quad i=2, \ldots, n .
$$

In addition, we design $u_{1}$ using the same construction of (32), specifically,

$$
u_{1}=-L^{n} \beta_{n}\left(\bar{\beta}_{n} \widehat{z}_{n}^{1 / r_{n}}+\bar{\beta}_{n-1} \widehat{z}_{n-1}^{1 / r_{n-1}}+\cdots+\bar{\beta}_{1} z_{1}\right)^{r_{n}+\tau} .
$$

Now, the closed-loop system (49)-(51) can be written as

$$
\dot{Z}=L F(Z)+\left(f_{1}(\cdot), \frac{f_{2}(\cdot)}{L}, \ldots, \frac{f_{n}(\cdot)}{L^{n-1}}, 0, \ldots, 0\right)^{T} .
$$

Hence, it can be concluded from (46) that

$$
\dot{U} \leq-L \bar{c}_{2}\|Z\|_{\Delta}^{2+\tau}+\frac{\partial U}{\partial Z}\left(f_{1}(\cdot), \frac{f_{2}(\cdot)}{L}, \ldots, \frac{f_{n}(\cdot)}{L^{n-1}}, 0, \ldots, 0\right)^{T} .
$$

From (21), (48), and $L \geq 1$, we can find constants $\delta_{i}>0$ and $\alpha_{i}<1$ such that

$$
\left|\frac{f_{i}(\cdot)}{L^{i-1}}\right| \leq b \sum_{j=1}^{i} \frac{L^{(j-1)\left(r_{i}+\tau\right) / r_{j}}}{L^{i-1}}\left|z_{j}\right|^{\left(r_{i}+\tau\right) / r_{j}} \leq \delta_{i} L^{\alpha_{i}}\|Z\|_{\Delta}^{r_{i}+\tau} .
$$

Noting that, for $i=1, \ldots, n, \partial U / \partial Z_{i}$ is homogeneous of degree $2-r_{i}$, we know that

$$
\frac{\partial U}{\partial Z_{i}}\left(\left|z_{1}\right|^{\left(r_{i}+\tau\right) / r_{1}}+\cdots+\left|z_{i}\right|^{\left(r_{i}+\tau\right) / r_{i}}\right)
$$

is homogeneous of degree $2+\tau$.

With (54) and (55) in mind, we can find a positive constant $c_{i}$ such that

$$
\left|\frac{\partial U}{\partial Z_{i}}\right|\left|\frac{f_{i}(\cdot)}{L^{i-1}}\right| \leq c_{i} L^{\alpha_{i}}\|Z\|_{\Delta}^{2+\tau}
$$

Substituting (56) into (53) yields

$$
\begin{aligned}
\dot{U} & \leq-L\left(\bar{c}_{2}-\sum_{i=1}^{n} c_{i} L^{\alpha_{i}-1}\right)\|Z\|_{\Delta}^{2+\tau} \\
& \leq-L\left(\bar{c}_{2}-\sum_{i=1}^{n} c_{i} L^{\alpha_{\max }-1}\right)\|Z\|_{\Delta}^{2+\tau},
\end{aligned}
$$

where $\alpha_{\max }=\max _{1 \leq i \leq n}\left\{\alpha_{i}\right\}<1$. Apparently, by choosing a large enough $L$, the right-hand side of (57) is negative definite.

Furthermore, it can be deduced from (57) that there is a constant $\bar{c}_{3}$ such that

$$
\dot{U} \leq-\bar{c}_{3} U^{(2+\tau) / 2} .
$$

By Lemma $6\left(U=V, c=\bar{c}_{3}\right.$, and $\left.\alpha=(2+\tau) / 2<1\right)$, (58) leads to the conclusion that the closed-loop system (20), (50), and (51) is globally finite-time stable, which yields that system (18) can be globally finite-time stabilized by the output feedback. In addition, the settling time $T_{1}$ satisfies

$$
T_{1} \leq \frac{-2 U^{(-\tau) / 2}(0)}{\bar{c}_{3} \tau}
$$

3.2. Finite-Time Output Feedback Stabilization of the $x_{0}$-Subsystem. From Section 3.1, we know that $x(t) \equiv 0$ when $t \geq$ $T_{1}$. Therefore, we just need to stabilize the $x_{0}$-subsystem in a finite time. When $t \geq T_{1}$, for the $x_{0}$-subsystem, we can take the following control law:

$$
\begin{gathered}
u_{0}\left(x_{0}\right)=g_{0}\left(x_{0}\right) x_{0}^{\alpha_{0}}, \quad 0<\alpha_{0}=\frac{\alpha_{1}}{\alpha_{2}}<1, \\
g_{0}\left(x_{0}\right)=-\frac{1}{c_{01}}\left(k_{0}+\phi_{0}\left(x_{0}\right)\right),
\end{gathered}
$$

where $k_{0}$ is a positive design constant, $\alpha_{i}, i=1,2$ are positive odd numbers, and $\phi_{0}\left(x_{0}\right) \geq a\left|x_{0}\right|^{1-\alpha_{0}} \geq 0$ is a smooth function. For instance, we can simply choose $\phi_{0}\left(x_{0}\right)=a\left(1+x_{0}^{2}\right)$.

Taking the Lyapunov function $V_{0}=x_{0}^{2} / 2$, a simple computation gives

$$
\dot{V}_{0} \leq-k_{0} x_{0}^{1+\alpha_{0}} \leq-k_{0} V_{0}^{\left(1+\alpha_{0}\right) / 2} .
$$

Thus, by Lemma $6, x_{0}$ tends to 0 within a settling time denoted by $T_{2}$ and

$$
T_{2} \leq \frac{2 V_{0}^{\left(1-\alpha_{0}\right) / 2}(0)}{k_{0}\left(1-\alpha_{0}\right)} .
$$

Up to now, we have finished the finite-time output feedback stabilizing controller design of the system (5). Consequently, the following theorem can be obtained to summarize the main result of the paper.

Theorem 20. Under Assumptions 1-3, if the proposed control design procedure together with the above switching control strategy is applied to system (5), then, for any initial conditions in the state space $\left(x_{0}, x\right) \in R^{n+1}$, the closed-loop system is globally finite-time regulated at origin. 


\section{Simulation Example}

To verify our proposed controller, we consider the following low-dimensional system:

$$
\begin{aligned}
& \dot{x}_{0}=d_{0} u_{0}+\theta_{0}(t) x_{0}, \\
& \dot{x}_{1}=d_{1} x_{2} u_{0}+\theta_{1}(t) \ln \left(1+x_{1}^{2}\right), \\
& \dot{x}_{2}=d_{2} u_{1}+\theta_{2} \sin x_{1},
\end{aligned}
$$

where $d_{i}, i=0,1,2$ are unknown constants and $\theta_{i}(t), i=$ $0,1,2$ are unknown functions.

It should be mentioned that, when $d_{1}=d_{2}=1$ and $\theta_{0}(t)=\theta_{1}(t)=\theta_{2}(t)=0$, the system (63) collapses into a third-order chained form system which can be viewed as the bilinear model of a mobile robot with small angle measurement error (see $[10,36]$ for more details). This means that the system (63) is a simple one; however, it comes from real world.

For simplicity, it is assumed that $d_{i} \in[0.5,1]$ and $\left|\theta_{i}(t)\right| \leq$ $1, i=1,2,3$. From this and Remark 4, it is not difficult to verify that Assumptions 1-3 hold. Firstly, we define the control law $u_{0}=1$ and introduce the change of coordinates

$$
\zeta_{1}=x_{1}, \quad \zeta_{2}=d_{1} x_{2}
$$

under which the $x$-subsystem of (63) is transformed into

$$
\begin{aligned}
& \dot{\zeta}_{1}=\zeta_{2}+\theta_{1}(t) \ln \left(1+\zeta_{1}^{2}\right), \\
& \dot{\zeta}_{2}=d_{1} d_{2} u_{1}+d_{1} \theta_{2}(t) \sin \zeta_{1} .
\end{aligned}
$$

If we pick $\tau=-2 / 5$, the dilation is defined as $r_{1}=1$ and $r_{2}=3 / 5$. Then, according to the design procedure shown in Section 3, we can explicitly construct an output feedback controller for system (65). We can choose specifically

$$
\begin{gathered}
\dot{\eta}_{2}=-L l_{1} \widehat{z}_{2}, \quad \widehat{z}_{2}=\left(\eta_{2}+l_{1} z_{1}\right)^{3 / 5}, \\
u_{1}=-L^{2} \beta_{2}\left(\widehat{z}_{2}^{5 / 3}+\beta_{1}^{5 / 3} z_{1}\right)^{1 / 5}
\end{gathered}
$$

with appropriate positive constants $l_{1}, \beta_{1}, \beta_{2}$, and a large enough gain $L$ such that output feedback controller (66) renders the system (65) (i.e., the $x$-subsystem of (63)) globally finite-time stable with a settling time $T_{1}$.

Then, when $t \geq T_{1}$, for the $x_{0}$-subsystem, we switch the control input $u_{0}$ to

$$
u_{0}\left(x_{0}\right)=-2 k_{0} x_{0}^{1 / 3}
$$

where $k_{0}$ is a positive design constant.

In the simulation, we assume $d_{0}=d_{1}=d_{2}=1$ and $\theta_{0}(t)=\theta_{1}(t)=\theta_{2}(t)=\sin t$. When $\left(x_{0}(0), x_{1}(0), x_{2}(0)\right.$, $\left.\eta_{2}(0)\right)=(0,1,-1,0)$, by choosing the gains for the output laws as $L=2, \beta_{1}=2, \beta_{2}=9, l_{1}=20$, and $k_{0}=1$, the simulation shown in Figure 1 demonstrates the global finite-time stability property of the closed-loop system (63)-(67).

Remark 21. Although system (63) was asymptotically stabilized by the existing output feedback controller in $[10,13]$, the system (66)-(67) is the first output feedback controller which globally finite-time stabilizes system (63). Compared to the existing asymptotical stabilization results, the proposed controller demonstrates more advantages such as faster convergence rates, higher accuracies, and better disturbance rejection properties [17].

\section{Conclusion}

This paper has solved the problem of global finite-time output feedback stabilization for a class of nonholonomic systems in chained form with uncertainties. With the help of backstepping recursive technique and the homogeneous domination approach, a constructive design procedure for output feedback control is given. It is shown that the designed control laws can guarantee that the closed-loop system states are globally finite-time regulated to zero. In this direction, there are still remaining problems to be investigated. For example, an interesting research problem is how to design a finite-time output feedback stabilizing controller for nonholonomic systems in stochastic setting.

\section{Appendix}

Proof of Proposition 14. By Lemma 9, one obtains

$$
\begin{aligned}
& -l_{i-1} e_{i}^{r_{i}}\left(z_{i}^{\left(2-r_{i-1}\right) / r_{i}}-\widehat{z}_{i}^{\left(2-r_{i-1}\right) / r_{i}}\right) \\
& =-l_{i-1} e_{i}^{r_{i}}\left(\left(z_{i}^{1 / r_{i}}\right)^{2-r_{i-1}}-\left(\widehat{z}_{i}^{1 / r_{i}}\right)^{2-r_{i-1}}\right) \\
& \leq-l_{i-1} \lambda_{i} e_{i}^{2+\tau},
\end{aligned}
$$

where $\lambda_{i}>0$ is a constant.

Proof of Proposition 15. Using $\gamma_{i}=\sigma_{i}+l_{i-1} z_{i-1}$, (26), (31), and Lemmas 9-11, it follows that

$$
\begin{aligned}
& \frac{2-r_{i-1}}{r_{i}} z_{i}^{\left(2-r_{i-1}-r_{i}\right) / r_{i}}\left(z_{i}^{\left(r_{i-1}\right) / r_{i}}-\gamma_{i}\right) z_{i+1} \\
&=\frac{2-r_{i-1}}{r_{i}}\left(\xi_{i+1}-\beta_{i}^{1 / r_{i+1}} \xi_{i}\right)^{r_{i+1}}\left(\xi_{i}-\beta_{i-1}^{1 / r_{i}} \xi_{i}\right)^{2-r_{i-1}-r_{i}} \\
& \times\left(\left(z_{i}^{r_{i-1} / r_{i}}-\widehat{z}_{i}^{r_{i-1} / r_{i}}\right)-l_{i-1}\left(z_{i-1}-\widehat{z}_{i-1}\right)\right) \\
& \leq k_{i 3}\left(\left|\xi_{i+1}\right|^{r_{i+1}}+\left|\xi_{i}\right|^{r_{i+1}}\right) \\
& \times\left(\left|\xi_{i}\right|^{2-r_{i-1}-r_{i}}+\left|\xi_{i-1}\right|^{2-r_{i-1}-r_{i}}\right) \\
& \times\left[\left|e_{i}\right|^{r_{i-1}}+\left|e_{i}\right|^{r_{i}}\left(\left|\xi_{i}\right|^{r_{i-1}-r_{i}}+\left|\xi_{i-1}\right|^{r_{i-1}-r_{i}}\right)\right. \\
& \leq\left.\frac{1}{12} \sum_{j=i-1}^{i+1} \xi_{j-1}^{2+\tau}+\left.e_{i-1}\right|^{r_{i-1}}\right]
\end{aligned}
$$

where $k_{i 3}>0, m_{i}>0$ are constants and $g_{i}$ is a continuous function of $l_{i-1}$. By $e_{1}=0$, one has $g_{2}=0$. 

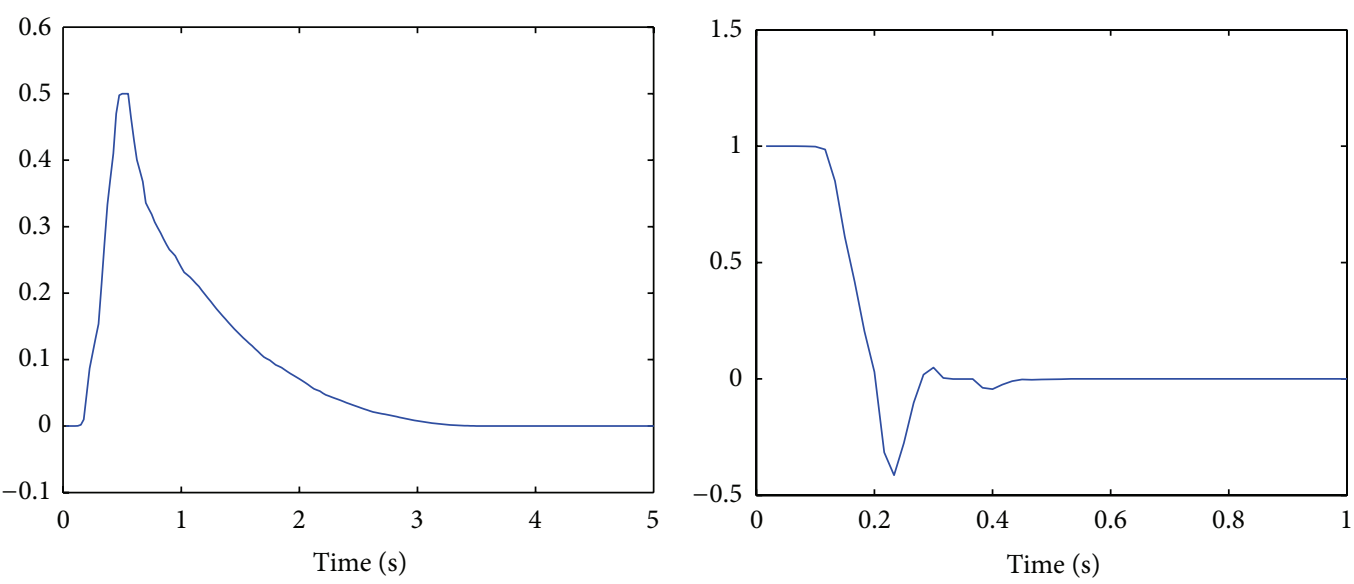

$-x_{0}$

$-x_{1}$

(a) $x_{0}$
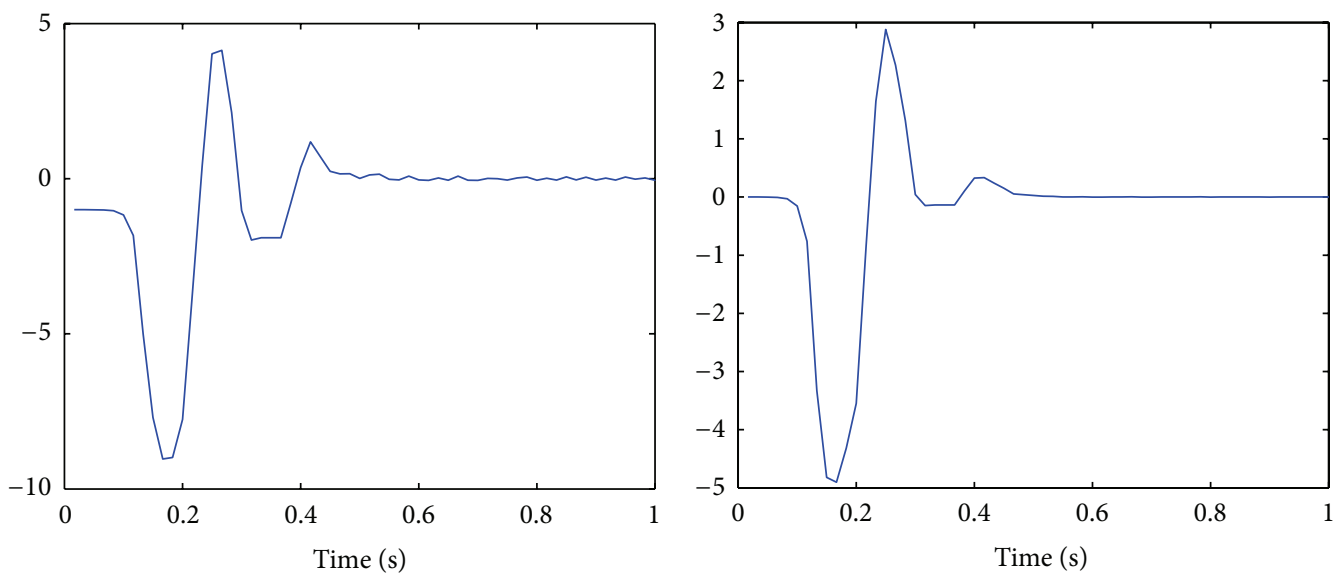

$-x_{2}$

$-\eta_{2}$

(c) $x_{2}$

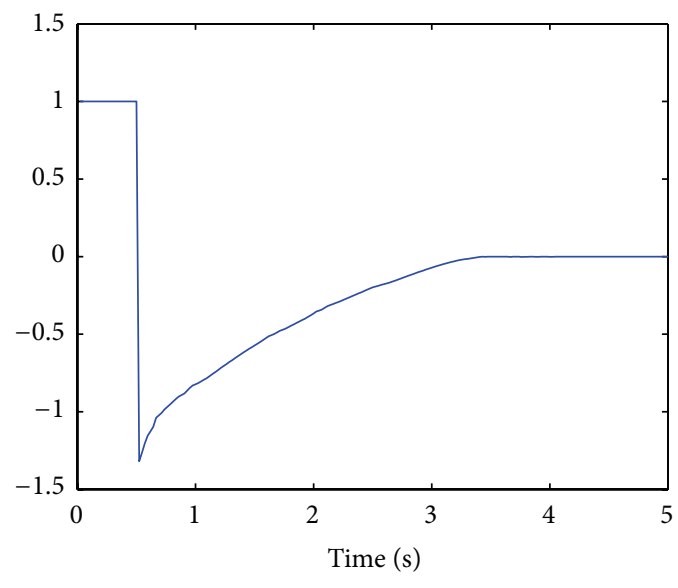

(d) $\eta_{2}$

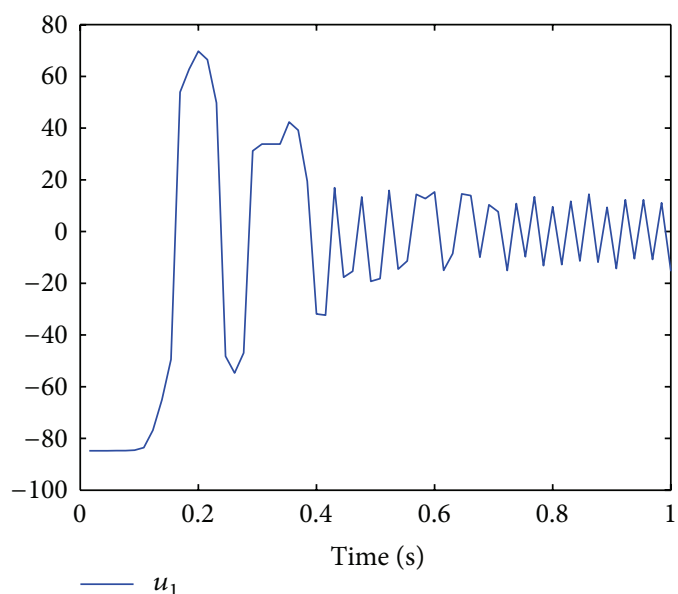

$-u_{0}$

(e) $u_{0}$

(f) $u_{1}$

FIgURE 1: The responses of the closed-loop system (63)-(67). 
Proof of Proposition 16. By (26), (32), $e_{i}=\left(z_{i}-\widehat{z}_{i}\right)^{1 / r_{i}}$, and the definition of the homogeneous norm, one gets

$$
\begin{aligned}
|v(\widehat{z})| & \leq k_{n 4}\|\widehat{z}\|_{\Delta}^{r_{n}+\tau} \\
& =k_{n 4}\left(\sum_{i=1}^{n}\left|\widehat{z}_{i}\right|^{2 / r_{i}}\right)^{\left(r_{n}+\tau\right) / 2} \\
& \leq \bar{k}_{n 4}\left(\sum_{i=1}^{n}\left|z_{i}\right|^{\left(r_{n}+\tau\right) / r_{i}}+\sum_{i=1}^{n}\left|e_{i}\right|^{r_{n}+\tau}\right) \\
& \leq \tilde{k}_{n 4}\left(\sum_{i=1}^{n}\left|\xi_{i}\right|^{r_{n}+\tau}+\sum_{i=1}^{n}\left|e_{i}\right|^{r_{n}+\tau}\right),
\end{aligned}
$$

where $k_{n 4}, \bar{k}_{n 4}$, and $\widetilde{k}_{n 4}$ are positive constants.

Similar to (A.2), with the use of Lemmas 9-11 and (A.3), (37) holds immediately.

Proof of Proposition 17. From $e_{i}=\left(z_{i}-\widehat{z}_{i}\right)^{1 / r_{i}},(26)$, and Lemma 9, there is a positive constant $k_{i 5}$ such that

$$
\left|\widehat{z}_{i}\right|=\left|z_{i}-e_{i}^{r_{i}}\right| \leq\left|z_{i}\right|+\left|e_{i}\right|^{r_{i}} \leq k_{i 5}\left(\left|\xi_{i-1}\right|^{r_{i}}+\left|\xi_{i}\right|^{r_{i}}+\left|e_{i}\right|^{r_{i}}\right) .
$$

According to $\gamma_{i}=\sigma_{i}+l_{i-1} z_{i-1}$, (31), (A.4), and Lemmas 9-11, one obtains

$$
\begin{aligned}
-l_{i-1} e_{i}^{r_{i}}\left(\widehat{z}_{i}^{\left(2-r_{i-1}\right) / r_{i}}-\gamma_{i}^{\left(2-r_{i-1}\right) / r_{i-1}}\right) & \\
= & l_{i-1} e_{i}^{r_{i}}\left(\left(\sigma_{i}+l_{i-1} \widehat{z}_{i-1}\right)^{\left(2-r_{i-1}\right) / r_{i-1}}\right. \\
\left.-\left(\sigma_{i}+l_{i-1} z_{i-1}\right)^{\left(2-r_{i-1}\right) / r_{i-1}}\right) & \\
\leq & k_{i 5} l_{i-1}^{2}\left|e_{i}\right|^{r_{i}}\left|e_{i-1}\right|^{r_{i-1}} \\
& \times\left(l_{i-1}^{\left(2-2 r_{i-1}\right) / r_{i-1}}\left|e_{i-1}\right|^{2-2 r_{i-1}}+\left|\xi_{i-1}\right|^{2-2 r_{i-1}}\right. \\
& \left.+\left|\xi_{i}\right|^{2-2 r_{i-1}}+\left|e_{i}\right|^{2-2 r_{i-1}}\right) \\
\leq & \frac{1}{16}\left(\xi_{i-1}^{2+\tau}+\xi_{i}^{2+\tau}\right)+e_{i}^{2+\tau}+h_{i}\left(l_{i-1}\right) e_{i-1}^{2+\tau},
\end{aligned}
$$

where $h_{i}$ is a continuous function of $l_{i-1}$.

Proof of Proposition 18. By (26), (31), and Lemmas 9-11, it follows that

$$
\begin{aligned}
& d \xi_{i}^{2-r_{n}}\left(v-z_{n+1}^{*}\right) \\
& =-d \xi_{i}^{2-r_{n}} \beta_{n}\left[\left(\bar{\beta}_{n} z_{n}^{1 / r_{n}}+\bar{\beta}_{n-1} z_{n-1}^{1 / r_{n-1}}+\cdots+\bar{\beta}_{1} z_{1}\right)^{r_{n}+\tau}\right. \\
& -\left(\bar{\beta}_{n} \hat{z}_{n}^{1 / r_{n}}+\bar{\beta}_{n-1} \widehat{z}_{n-1}^{1 / r_{n-1}}\right. \\
& \left.\left.+\cdots+\bar{\beta}_{1} z_{1}\right)^{r_{n}+\tau}\right] \\
& \leq k_{n 6}\left|\xi_{i}\right|^{2-r_{n}} \\
& \times\left[\sum_{i=2}^{n}\left|z_{i}-\widehat{z}_{i}\right|\left(\left|z_{i}-\widehat{z}_{i}\right|^{\left(1-r_{i}\right) / r_{i}}+\left|z_{i}\right|^{\left(1-r_{i}\right) / r_{i}}\right)\right]^{r_{n}+\tau}
\end{aligned}
$$

$$
\begin{aligned}
= & \bar{k}_{n 6}\left|\xi_{i}\right|^{2-r_{n}} \\
& \times\left[\sum_{i=2}^{n}\left|e_{i}\right|^{r_{i}}\left(\left|e_{i}\right|^{1-r_{i}}+\left|\xi_{i-1}\right|^{1-r_{i}}+\left|\xi_{i}\right|^{1-r_{i}}\right)\right]^{r_{n}+\tau} \\
\leq & \frac{1}{4} \sum_{i=1}^{n} \xi_{i}^{2+\tau}+\mu \sum_{i=2}^{n} e_{i}^{2+\tau}
\end{aligned}
$$

where $k_{n 6}, \bar{k}_{n 6}$, and $\mu$ are positive constants.

\section{Acknowledgments}

The authors would like to thank the editor and the anonymous reviewers for their constructive comments and suggestions for improving the quality of the paper. This work has been supported in part by the National Natural Science Foundation of China under Grant 61073065 and the Key Program of Science Technology Research of Education Department of Henan Province under Grant 13A120016.

\section{References}

[1] R. W. Brockett, "Asymptotic stability and feedback stabilization," in Differential Geometric Control Theory, R. W. Brockett, R. S. Millman, and H. J. Sussmann, Eds., pp. 2961-2963, 1983.

[2] A. Astolfi, "Discontinuous control of nonholonomic systems," Systems \& Control Letters, vol. 27, no. 1, pp. 37-45, 1996.

[3] W. L. Xu and W. Huo, "Variable structure exponential stabilization of chained systems based on the extended nonholonomic integrator," Systems \& Control Letters, vol. 41, no. 4, pp. 225-235, 2000.

[4] R. M. Murray and S. S. Sastry, "Nonholonomic motion planning: steering using sinusoids," IEEE Transactions on Automatic Control, vol. 38, no. 5, pp. 700-716, 1993.

[5] Z.-P. Jiang, "Iterative design of time-varying stabilizers for multi-input systems in chained form," Systems \& Control Letters, vol. 28, no. 5, pp. 255-262, 1996.

[6] Y.-P. Tian and S. Li, "Exponential stabilization of nonholonomic dynamic systems by smooth time-varying control," Automatica, vol. 38, no. 7, pp. 1139-1146, 2002.

[7] G. C. Walsh and L. G. Bushnell, "Stabilization of multiple input chained form control systems," Systems \& Control Letters, vol. 25, no. 3, pp. 227-234, 1995.

[8] J. P. Hespanha, D. Liberzon, and A. S. Morse, "Towards the supervisory control of uncertain nonholonomic systems," in Proceedings of the American Control Conference (ACC '99), pp. 3520-3524, San Diego, Calif, USA, June 1999.

[9] Z.-Y. Liang and C.-L. Wang, "Robust stabilization of nonholonomic chained form systems with uncertainties," Acta Automatica Sinica, vol. 37, no. 2, pp. 129-142, 2011.

[10] Z.-P. Jiang, "Robust exponential regulation of nonholonomic systems with uncertainties," Automatica, vol. 36, no. 2, pp. 189209, 2000.

[11] S. S. Ge, Z. Wang, and T. H. Lee, "Adaptive stabilization of uncertain nonholonomic systems by state and output feedback," Automatica, vol. 39, no. 8, pp. 1451-1460, 2003.

[12] Y.-G. Liu and J.-F. Zhang, "Output-feedback adaptive stabilization control design for non-holonomic systems with strong 
non-linear drifts," International Journal of Control, vol. 78, no. 7, pp. 474-490, 2005.

[13] Z. Xi, G. Feng, Z. P. Jiang, and D. Cheng, "Output feedback exponential stabilization of uncertain chained systems," Journal of the Franklin Institute, vol. 344, no. 1, pp. 36-57, 2007.

[14] X. Zheng and Y. Wu, "Adaptive output feedback stabilization for nonholonomic systems with strong nonlinear drifts," Nonlinear Analysis: Theory, Methods \& Applications, vol. 70, no. 2, pp. 904920, 2009.

[15] G. Ju, Y. Wu, and W. Sun, "Output-feedback control for nonholonomic systems with linear growth condition," Journal of Systems Science \& Complexity, vol. 24, no. 5, pp. 862-874, 2011.

[16] Y. Zhao and Y. Q. Wu, "Control of nonholonomic systems with nonlinear unmeasured dynamics by output feedback," Journal of Control Theory and Applications, vol. 11, no. 3, pp. 504-512, 2013.

[17] S. P. Bhat and D. S. Bernstein, "Finite-time stability of continuous autonomous systems," SIAM Journal on Control and Optimization, vol. 38, no. 3, pp. 751-766, 2000.

[18] Y. Hong, "Finite-time stabilization and stabilizability of a class of controllable systems," Systems \& Control Letters, vol. 46, no. 4, pp. 231-236, 2002.

[19] Y. Hong, J. Huang, and Y. Xu, "On an output feedback finitetime stabilization problem," IEEE Transactions on Automatic Control, vol. 46, no. 2, pp. 305-309, 2001.

[20] X. Huang, W. Lin, and B. Yang, "Global finite-time stabilization of a class of uncertain nonlinear systems," Automatica, vol. 41, no. 5, pp. 881-888, 2005.

[21] Y. Hong, J. Wang, and D. Cheng, "Adaptive finite-time control of nonlinear systems with parametric uncertainty," IEEE Transactions on Automatic Control, vol. 51, no. 5, pp. 858-862, 2006.

[22] J. Li and C. Qian, "Global finite-time stabilization by dynamic output feedback for a class of continuous nonlinear systems," IEEE Transactions on Automatic Control, vol. 51, no. 5, pp. 879884, 2006.

[23] J. Li, C. Qian, and S. Ding, "Global finite-time stabilisation by output feedback for a class of uncertain nonlinear systems," International Journal of Control, vol. 83, no. 11, pp. 2241-2252, 2010.

[24] Y. Shen and Y. Huang, "Global finite-time stabilisation for a class of nonlinear systems," International Journal of Systems Science, vol. 43, no. 1, pp. 73-78, 2012.

[25] J. Y. Zhai, "Global finite-time output feedback stabilisation for a class of uncertain nontriangular nonlinear systems," International Journal of Systems Science, vol. 45, no. 3, pp. 637-646, 2012.

[26] Y. Hong, J. Wang, and Z. Xi, "Stabilization of uncertain chained form systems within finite settling time," IEEE Transactions on Automatic Control, vol. 50, no. 9, pp. 1379-1384, 2005.

[27] J. Wang, G. Zhang, and H. Li, "Adaptive control of uncertain nonholonomic systems in finite time," Kybernetika, vol. 45, no. 5, pp. 809-824, 2009.

[28] F. Gao, Y. Shang, and F. Yuan, "Robust adaptive finite-time stabilization of nonlinearly parameterized nonholonomic systems," Acta Applicandae Mathematicae, vol.123, no. 1, pp. 157-173, 2013.

[29] H. Hermes, "Homogeneous coordinates and continuous asymptotically stabilizing feedback controls," in Differential Equations, vol. 127, pp. 249-260, Dekker, New York, NY, USA, 1991.

[30] A. Bacciotti and L. Rosier, Liapunov Functions and Stability in Control Theory, Springer, Berlin, Germany, 2005.
[31] J. Polendo and C. Qian, "A generalized homogeneous domination approach for global stabilization of inherently nonlinear systems via output feedback," International Journal of Robust and Nonlinear Control, vol. 17, no. 7, pp. 605-629, 2007.

[32] C. Qian and W. Lin, "A continuous feedback approach to global strong stabilization of nonlinear systems," IEEE Transactions on Automatic Control, vol. 46, no. 7, pp. 1061-1079, 2001.

[33] J. Polendo and C. Qian, "A generalized framework for global output feedback stabilization of genuinely nonlinear systems," in Proceedings of the 44th IEEE Conference on Decision and Control, and the European Control Conference (CDC-ECC '05), pp. 2646-2651, December 2005.

[34] C. Qian, "A homogeneous domination approach for global output feedback stabilization of a class of nonlinear systems," in Proceedings of the American Control Conference (ACC '5), pp. 4708-4715, June 2005.

[35] J. Kurzweil, “On the inversion of Lyapunov's second theorem on the stability of motion," Annals of Mathematical Society Translations, vol. 24, no. 2, pp. 19-77, 1956.

[36] P. Morin, J. B. Pomet, and C. Samson, "Developments in time varying feedback stabilization of nonlinear systems," in Preprints of Nonlinear Control Systems Design Symposium, pp. 587-594, Enschede, The Netherlands, 1998. 


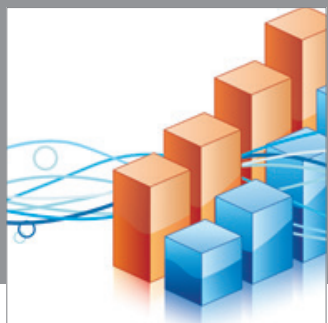

Advances in

Operations Research

mansans

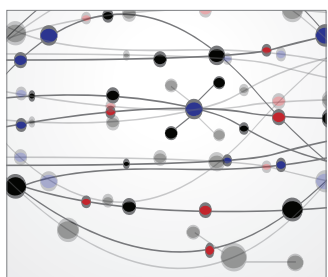

The Scientific World Journal
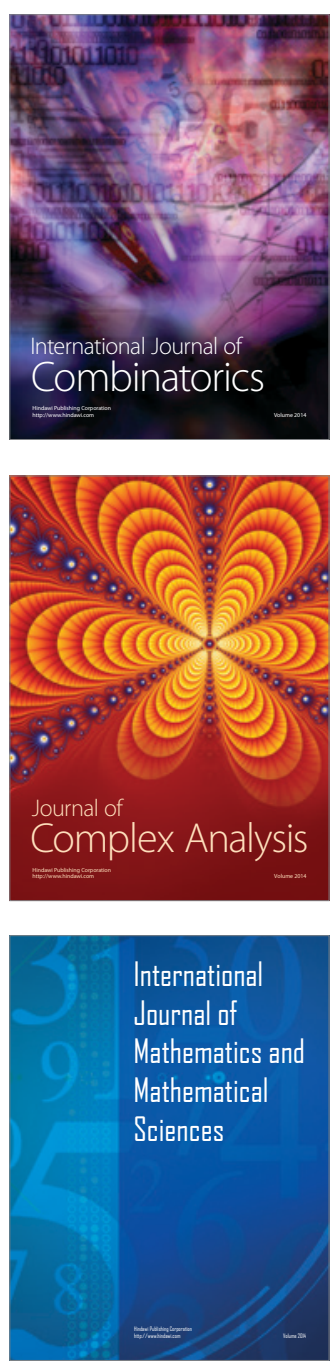
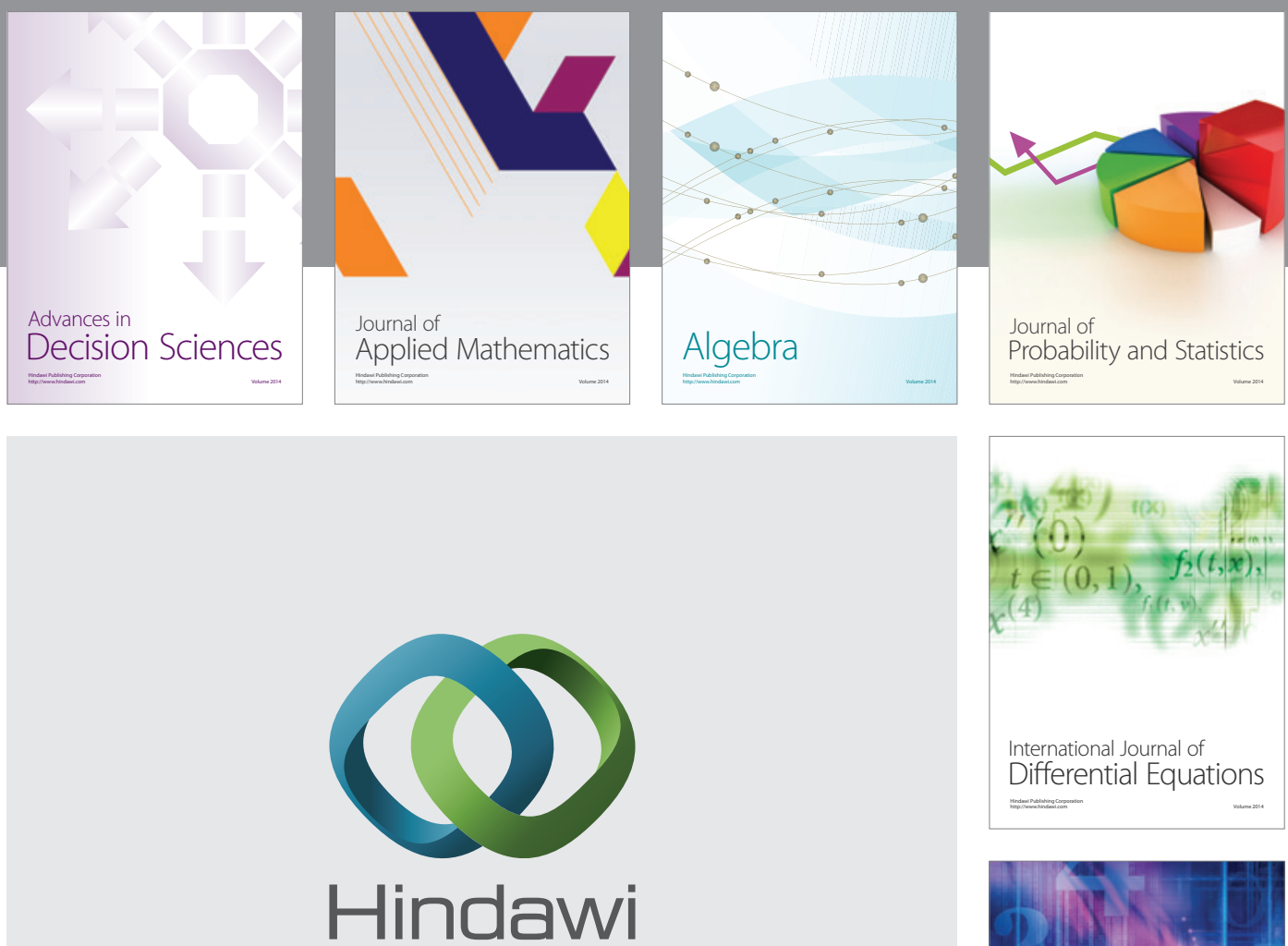

Submit your manuscripts at http://www.hindawi.com
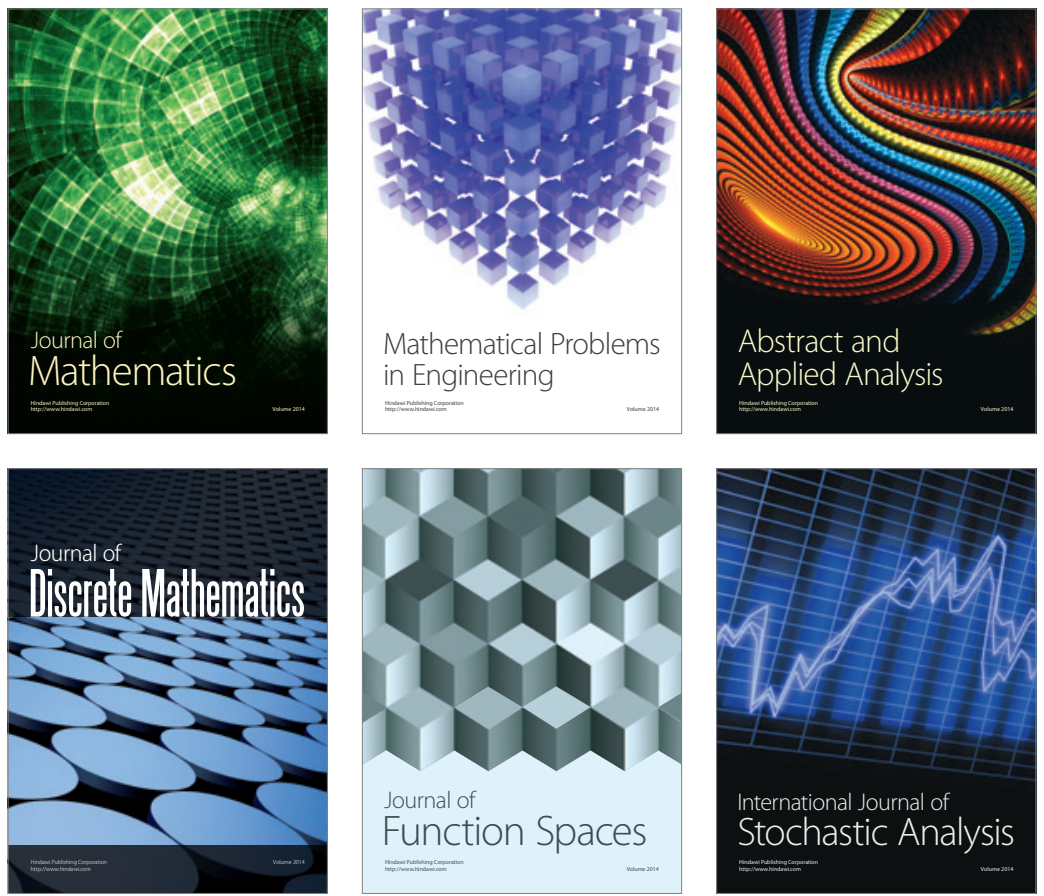

Journal of

Function Spaces

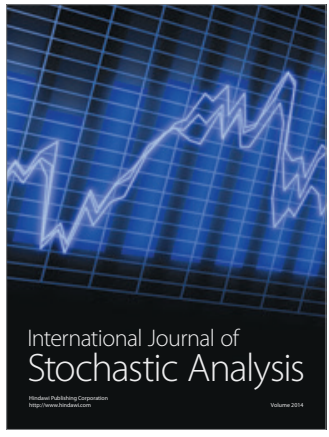

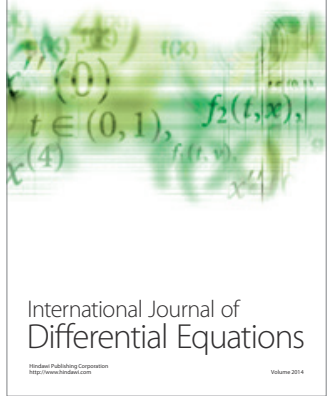
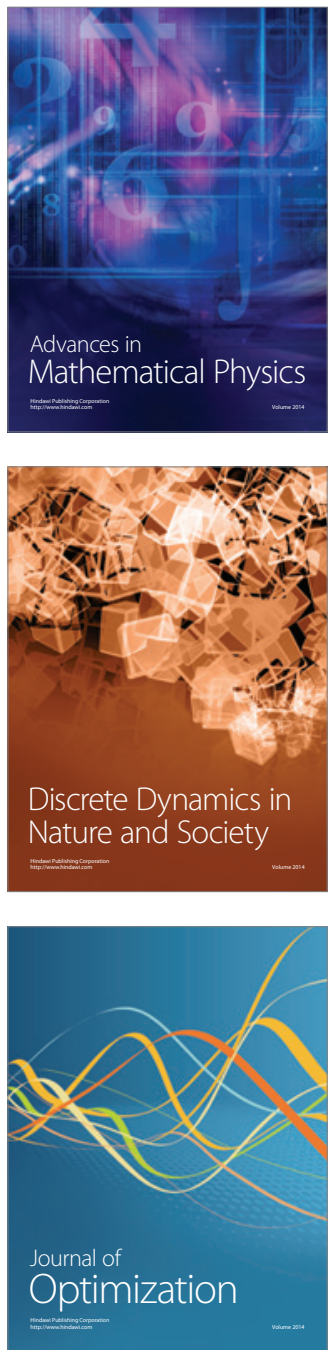